E. Arribas, I. Escobar, C. P. Suárez, A. Najera and A. Beléndez, "Measurement of the magnetic field of small magnets with a smartphone: a very economical laboratory practice for introductory physics courses", European Journal of Physics Vol. 36, No. 6, Art. ID 065002, 11 pp (2015).

\title{
Measurement of the magnetic field of small magnets with a smartphone: a very economical laboratory practice for introductory physics courses
}

\section{Enrique Arribas}

Applied Physics Department, High School of Informatics Engineering, University of Castilla-La Mancha, Albacete, Spain

\section{Isabel Escobar}

Applied Physics Department, Polytechnic School of Cuenca, University of Castilla-La Mancha, Cuenca

\section{Carmen P. Suárez}

Academic Coordination Huasteca South, Autonomous University of San Luis Potosí, Tamazunchale San Luis Potosí, México

\author{
Alberto Nájera \\ Medical Science Department, School of Medicine, University of Castilla-La Mancha, Albacete, Spain

\section{Augusto Beléndez} \\ Department of Physics, Systems Engineering and Signal Theory, University of Alicante, Alicante, Spain
}

\begin{abstract}
In this work we propose an inexpensive laboratory practice for the laboratory of an introductory course of Physics for any grade of Sciences and Engineering, which was very well received by our students, where a smartphone (iOS, Android or Windows Phone is irrelevant) is used together with some mini magnets, similar to those that can be found in the door of our refrigerators, a $20 \mathrm{~cm}$ long school rule, a paper and a free application (app) for measuring the magnetic field using the magnetic fields sensor or magnetometer of the smartphone, which needs to be downloaded and installed. The apps we have used are: Magnetometer (iOS), Magnetometer Metal Detector and Physics Toolbox Magnetometer (Android). Nothing else is needed. Cost of this practice: 0 coins. The main purpose of the practice is that students determine the dependence of the component $\mathrm{x}$ of the magnetic field produced by different magnets (from the typical magnets that are decorated in refrigerators even with a ring magnet and spherical magnet). We have obtained that the dependency of the magnetic field with the distance is of the form $\mathrm{x}^{-3}$, in total agreement with the theoretical analysis. The secondary objective is to apply the technique of least squares fit to obtain this exponent and the magnetic moment of the magnets, with theirs corresponding absolute error.
\end{abstract}

Keywords: university physics, lab physics, new technologies, active learning, experimental, smartphone. 
E. Arribas, I. Escobar, C. P. Suárez, A. Najera and A. Beléndez, "Measurement of the magnetic field of small magnets with a smartphone: a very economical laboratory practice for introductory physics courses", European Journal of Physics Vol. 36, No. 6, Art. ID 065002, 11 pp (2015).

\section{INTRODUCTION}

Meaningful learning of Physics in the first college courses in Science and Engineering results, in many occasions, relatively complex. In addition to the theoretical classes and problems, it is necessary for students to carry out laboratory practice, because we are convinced that the words of Benjamin Franklin: "Tell me and I forget, teach me and I may remember, involve me and I learn" continue to be paradigmatic in Physics teaching at any level of education, and especially at the university. According to Vygotsky ${ }^{1,2}$, the practical activity is conducive to promote the development of higher mental processes which are very characteristic of human beings, this allows the generation and acquisition of constructivist knowledge that is developed favourably through the participation of the subject in cooperative group processes and in the exchange of ideas encouraging the acquisition of knowledge.

As we well know, Physics (along with other sciences) is different from other subjects, because, most of the time, the answers to the questions we can do to ourselves are not found in books, but they are accomplished through reasoning and inferences, which become sometimes laborious. The knowledge of physicists comes from the observation of the evidence obtained in a laboratory and a subsequent detailed analysis. The exception might be theoretical physics and its beautiful theories, some so difficult to verify; for instance the history of the boson Higgs: forty years after his theoretical prediction its existence was experimentally confirmed, although in a very complex and indirect manner.

The laboratory is almost "sacred", it is the place where Physics (with capital letters) is made. In there, the students learn (or should learn) to make themselves questions, to follow protocols, to answer doubts and to germinate new questions in their minds which allows them to explore the knowledge in a more autonomous way. The assistance itself to the laboratory, the capture of information, its note in a notebook or in a portable computer, the precise acquisition and treatment of the experimental information, its adjustment and later interpretation, the calculation of errors and the achievement of an auto sufficient graph, are tasks that our students must learn and perform by working, normally, in groups of two. The achievement of the laboratory experiments does not only allow to acquire an experience -different and complementary to the acquired in the theoretical classes and in the solution of problems -on the phenomena, laws and theories of Physics, but also increases the skills on the synthesis of the ideas and the ability to translate it into writing in a memory. This provides our students with the opportunity to familiarize with the measuring devices, to breathe in an atmosphere of scientific work, similar although on a different scale- to the one in a research laboratory. The physics laboratory is a vital complement to the theoretical classes so that students are involved in their own learning in a highly significant manner. We can say that the laboratory classes are those teaching activities in which the student is the main character.

We are advocates that students should perform accurate measurements using different laboratory devices: ammeter, voltmeter, capacitance meter, etc. However, and although we are not against the practices of an automated laboratory in which through an interface the measurements go directly to a computer, we are of the opinion that these last ones are perhaps more interesting for advanced courses than for introductory physics courses, since we intend that the student on the freshmen university courses be more involved in data collection. Physics 
E. Arribas, I. Escobar, C. P. Suárez, A. Najera and A. Beléndez, "Measurement of the magnetic field of small magnets with a smartphone: a very economical laboratory practice for introductory physics courses", European Journal of Physics Vol. 36, No. 6, Art. ID 065002, 11 pp (2015).

laboratories are being filled more and more with sophisticated and expensive practices, which leads to having fewer jobs -sometimes there is only one position which is also used by the teacher himself/herself to make a demonstration in class- and, which is more important, the need to make a significant economic investment, which in many technical schools and university faculties is very difficult.

Since many years ago the portable devices are colonizing almost all the levels of education, both university and not university. Numerous investigations have been published on laboratory experiments in which digital cameras or webcams are used ${ }^{3-7}$, optical mice ${ }^{8,9}$ and games consoles and their wireless devices which control the games ${ }^{10-14}$. Also the sensors of smartphones are beginning to be used to make precise measurements ${ }^{15-20}$. These sensors allow carrying out direct measurements of physical magnitudes if we have previously downloaded specific applications, most of which are free, that allow us to show measurements on the screen of the smartphone, store them and even do a graph with the results.

Bearing this in mind, in this work we are going to centre on the use of the magnetic sensor of smartphones to measure the dependence on the magnetic field produced by a magnet in function of the distance to the same one, and compare the results with the theoretical predictions.

\section{MATERIAL AND MOTIVATION}

In this work, we propose a very cheap laboratory practice and which has been very well accepted by our students. What do we need? A smartphone for every two students (iOS, Android or Windows Phone, is indifferent) a small magnet, those we have stuck on the door of our refrigerators, a $20 \mathrm{~cm}$ school rule, a paper and a pencil. We don't need anything else. Taking into account that today it is difficult to find a student without a smartphone, we can affirm that the cost of this practice of laboratory is 0 coins.

The penetration of the last generation cell phones (smartphone or smart phones) amongst our students round a percentage close to $100 \%$, in addition to this, they are perhaps the greatest experts in using them, certainly much more than teachers. We believe that it is correct to use a smartphone as a new tool in the laboratory for several reasons: they are tremendously motivators, they are very versatile, they are filled with sensors that if properly used they allow instant direct measures and in addition students feel a special attraction for new technologies, that interest can be taken advantage of, indirectly, so students can learn Physics.

\section{BASIC THEORY}

The $x$ component of the magnetic field created by a small magnet of length $d$ and magnetic moment with module $m$ at a point located at a distance $x$ along the axis of the magnet (which coincides with the direction of the magnetic moment of the magnet) is given by the equation (see Fig. 1) ${ }^{21}$,

$$
B=\frac{\mu_{0} m x}{2 \pi\left(x^{2}-d^{2} / 4\right)^{2}}
$$


E. Arribas, I. Escobar, C. P. Suárez, A. Najera and A. Beléndez, "Measurement of the magnetic field of small magnets with a smartphone: a very economical laboratory practice for introductory physics courses", European Journal of Physics Vol. 36, No. 6, Art. ID 065002, 11 pp (2015).

being $\mu_{0}$ the magnetic permeability of the free space $\left(\mu_{0}=4 \pi \cdot 10^{-7} \mathrm{~N} / \mathrm{A}^{2} \mathrm{o} \mathrm{H} / \mathrm{m}\right)$. In the SI, the units of the magnetic moment of a magnet, $m$, are $\mathrm{A} \cdot \mathrm{m}^{2} \mathrm{o} \mathrm{J} / \mathrm{T}$. If we evaluate this magnetic field at a distance $x$, much larger than the size of the magnet, $d$, we can simplify the previous result taking into account that for $x>d$ the previous expression takes the form of

$$
B=\frac{\mu_{0} m}{2 \pi x^{3}}
$$

In this way, it is obtained that the $x$ component of the magnetic field of a magnetic dipole is proportional to $x^{-3}$, appearing in the proportionality constant the magnetic permeability of the vacuum, $\mu_{0}$, and the magnetic moment of the magnet, $m$. With these mathematical tools we are going to proceed to measure the $x$ component of the magnetic field of a small magnet in function of the distance to the centre of the magnet using a smartphone ${ }^{21-24}$.

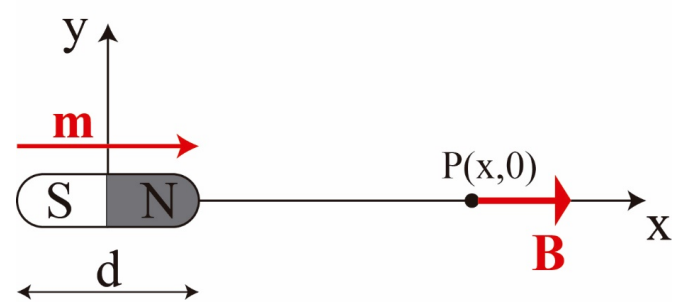

Figure 1. Small magnet of length $d$ and magnetic moment with module $m$ placed on the $\mathrm{X}$-axis centered at the coordinate origin, and the $x$ component of the magnetic field at a point $\mathrm{P}$ over the $\mathrm{X}$-axis.

\section{METHODOLOGY}

To carry out this laboratory practice we need to have an application that measures the magnetic field installed on our smartphone. It is important to install an application that is able to determine the three spatial components of the magnetic field, not only its module, because with the module is not sufficient. On the Internet, there are different apps that allow making these measures and we recommend "Magnetometer". In Fig. 2 we have displayed a screenshot of this app running on smartphones with an operating system $\mathrm{iOS}^{25}$. Other interesting apps are "Magnetometer Metal Detector" and "Physics Toolbox Magnetometer" that can be used on smartphones with Android operating system ${ }^{26}$.

These apps often have a settings screen and in it, it appears the update frequency reading that is usually between $2-10 \mathrm{~Hz}$. For high values, it is difficult to read the decimal part because it varies very quickly, due to the fact that the app takes a data from the sensor every tenth of a second. If we adjust it to $2 \mathrm{~Hz}$, it takes a data every half-second and it is easier to write down, both the whole part and the first decimal digit. It is not needed to have more precision, whenever we take three measurements (at least) and we calculate the arithmetic mean, which is better than any of them. 
E. Arribas, I. Escobar, C. P. Suárez, A. Najera and A. Beléndez, "Measurement of the magnetic field of small magnets with a smartphone: a very economical laboratory practice for introductory physics courses", European Journal of Physics Vol. 36, No. 6, Art. ID 065002, 11 pp (2015).

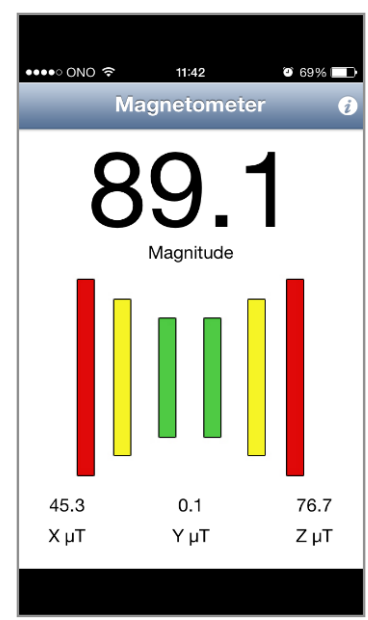

Figure 2. Screenshot of the Magnetometer App that allows determining the three components and the module of magnetic field from a small magnet.

Since the objective of the practice is to determine the dependency of the magnetic field with the distance, we will consider only a component of the magnetic field, for example the $x$ component. But, how do we know the availability of the XYZ axes in our smartphone? It takes only a small discovery process which consists to bring near a small magnet (the ones we use on the refrigerators) to our phone in different directions and observe the component that varies. In this way, we can determine the direction of the XYZ Cartesian axes, which are oriented as shown in Fig. 3.

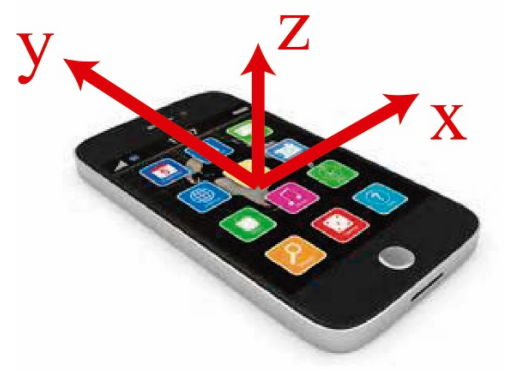

Figure 3. Orientation of the spatial axes on a smartphone

Another relevant aspect to take into account is the position of the detector in the interior of the smartphone. Once again, and on an experimental basis, we are moving the magnet slowly on the phone screen and noting the position in which the module of magnetic field is higher. At that point exactly, within the smartphone, the magnetic sensor will be located. In Fig 4 it is shown, as an example, the position of the magnetic detector in an iPhone 5. That distance must be added then to the $x$ variable. 
E. Arribas, I. Escobar, C. P. Suárez, A. Najera and A. Beléndez, "Measurement of the magnetic field of small magnets with a smartphone: a very economical laboratory practice for introductory physics courses", European Journal of Physics Vol. 36, No. 6, Art. ID 065002, 11 pp (2015).

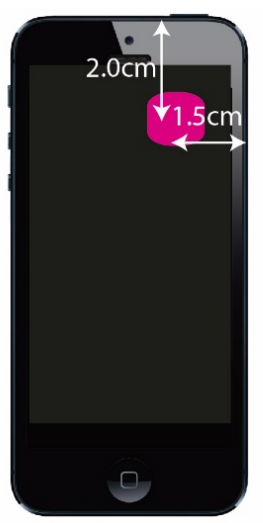

Figure 4. Location of the magnetic sensor in the interior of an iPhone 5.

As we well know, the earth itself is a magnet. Its geographical North Pole is relatively close to the magnetic South Pole, which is the reason why the North Pole of a compass needle points to the geographical North of the earth. We also know that the Earth's magnetic field is of the order of $50 \mu \mathrm{T}$. The magnetic field produced by the laboratory magnets lies in the range $\mu \mathrm{T}-\mathrm{mT}$, depending on the distance from the detector to the magnet. From these data, we can conclude that the Earth's magnetic field will influence in our measurements due to the fact that they are similar or superior orders of magnitude. To avoid having a background of the magnetic field coming from the terrestrial magnetic field, the only thing we have to do is to direct our smartphone in a particular position when making measurements. Experimentally, we will turn the smartphone slowly until we detect a position in which the value of the component of the magnetic field $x$ is practically null. If we do not manage to display the value 0 we must enter the minimum value (the average of three observations) and we will call them $B_{x 0}$, later we will have to subtract or add from our measures in function of it being positive or negative, respectively. If we analyse this position, we will realize that the phone has just stayed oriented in N-S direction, and therefore, the $x$ axis is perpendicular to such direction, as shown in Fig. 3 .

Once we have taken into account all these preliminary adjustments, we can perform the experimental assembly of the practice, which is very simple, as shown in Fig. 5, we place our smartphone at a suitable orientation on a sheet of paper size DIN A4 and draw the corresponding axis $x$ of the phone that must pass through the sensor. Then, we will be placing a magnet at different distances and write down the value of the $x$ component of the magnetic field provided by the downloaded application. It should be recalled, taking into account Figure 4 that we have to add the distance to which the magnetometer is located from the coordinate origin, because this sensor is inside the smartphone (1.5 $\mathrm{cm}$ for an iPhone 5$)$. 
E. Arribas, I. Escobar, C. P. Suárez, A. Najera and A. Beléndez, "Measurement of the magnetic field of small magnets with a smartphone: a very economical laboratory practice for introductory physics courses", European Journal of Physics Vol. 36, No. 6, Art. ID 065002, 11 pp (2015).

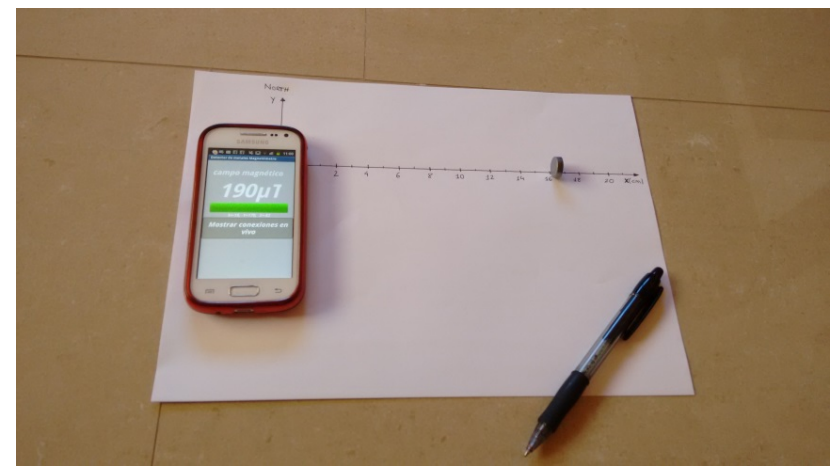

Figure 5. Experimental assembly in which you can perceive the small magnet and the smartphone with its head oriented towards the geographic North (South magnetic).

\section{RESULTS}

Next, we will analyse the results that were obtained with different models of smartphones and with magnets of different forms and magnetic moments, from the typical magnets that are used as decorations on refrigerators even with a ring magnet (see Fig. 6).

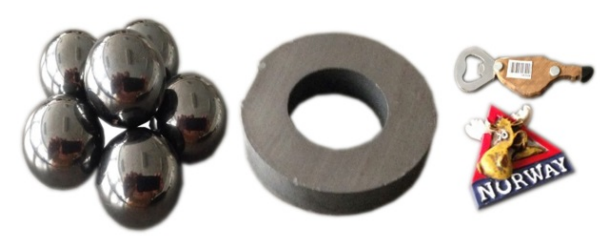

Figure 6. Diverse magnets used: spheres, ring and two typical refrigerator magnets.

For the development of the experience, first it is proposed to the students the following equation for the $x$ component of the magnetic field of the Dipole:

$$
B=\frac{\mu_{0} m x^{n}}{2 \pi}
$$

So they must determine the value of $n$ from the experimental data. If the theoretical model (Eq. 2) is correct, the value that is obtained for $n$ should be approximately -3 .

Fig. 7 shows the graphical representation of the data taken with the magnetic sensor of the smartphone for the component $x$ of the magnetic field $B$ in function of the $x$ distance, for the two less powerful magnets case, the typical ones which are usually attached to the refrigerators doors as ornaments. In this figure it has also been included the setting of the experimental data with EXCEL, using the option "potential adjustment". You can see the curve of adjustment, the 
E. Arribas, I. Escobar, C. P. Suárez, A. Najera and A. Beléndez, "Measurement of the magnetic field of small magnets with a smartphone: a very economical laboratory practice for introductory physics courses", European Journal of Physics Vol. 36, No. 6, Art. ID 065002, 11 pp (2015).

equation that adjusts the experimental data ( $B$ as a function of $x$ ) and the correlation coefficient, $R$, square.

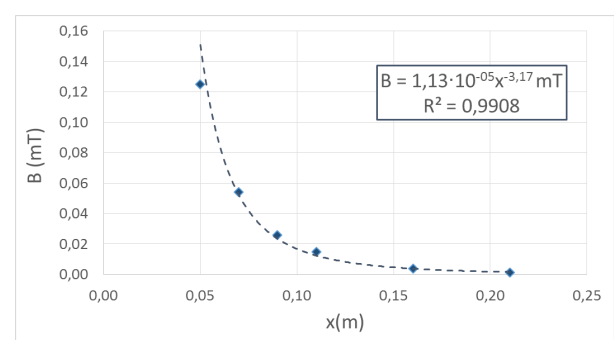

a)

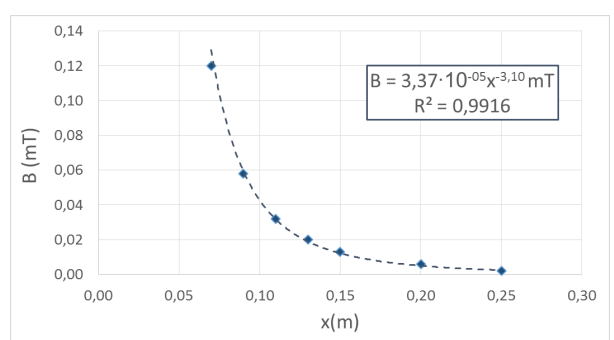

b)

Figure 7. Experimental measures $(\bullet)$ of the magnetic field of a refrigerator magnet using the sensor of the smarthphone and potential adjustment of the experimental measures (---) by two different smartphones; a) Sony Xperia Play with the Physics Toolbox, Magnetometer function application; b) Jiayu G3 with the Physics Toolbox, Magnetometer function application.

Fig. 8 shows the same results as in Fig. 7 but for two more powerful magnets, one spherical and the other ring shaped. For the spherical, it is necessary to previously find out where its North and South poles are.

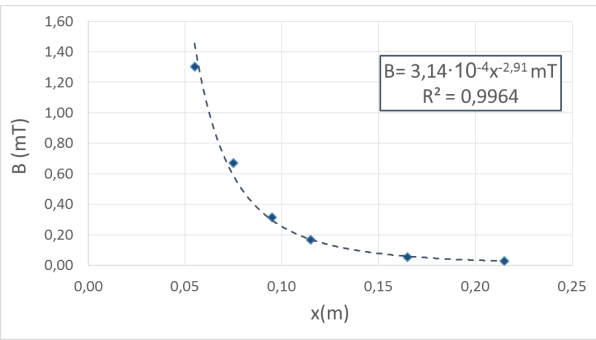

a)

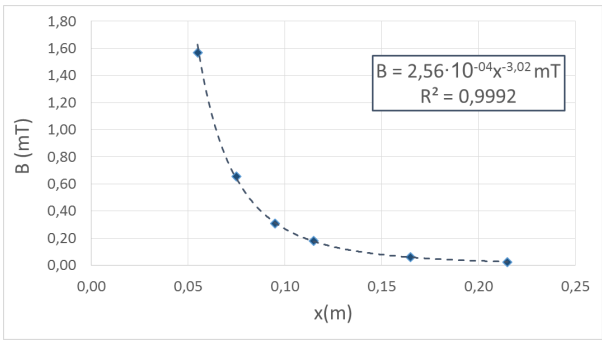

b)

Figure 8. Experimental measures $(\diamond)$ of the magnetic field on magnets somewhat more powerful using the sensor of the smarthphone and a potential adjustment of the experimental measures (---) for the following special cases we used: a) spherical magnet and a Samsung Galaxy Ace 2 Smartphone, Magnetometer Metal Detector application; (b) Ring magnet and an iPhone 5 smartphone with the Magnetometer application.

Based on the results of the Figs. 7 and 8, it is possible to observe that the four measurements with different smartphones, have a very high square correlation close to the unit, being the lowest 0.9908 (fridge magnet) and the highest 0.9992 (ring shaped magnet), much higher than the minimum that we require in the laboratory, 0.95 . 
E. Arribas, I. Escobar, C. P. Suárez, A. Najera and A. Beléndez, "Measurement of the magnetic field of small magnets with a smartphone: a very economical laboratory practice for introductory physics courses", European Journal of Physics Vol. 36, No. 6, Art. ID 065002 , 11 pp (2015).

Also, we observed that the values of $n$ that we have obtained vary between - 3.17 (fridge magnet) and -2.91 (spherical magnet), which tells us that the dependency of the magnetic field with the distance is of the form $x^{-3}$, in total agreement with the theoretical analysis (Eq. 2).

At this point, for many students it is one sufficient objective to be aware that the exponent $n$ is very close to -3 . However, there is close to $25 \%$ of the total number of students who can, wish to and are ready to carry out more elaborated calculations and obtain new results. To these students, we proposed to treat the same experimental measures that have been used to determine the value of $n$ in an alternative way, through the adjustment of a straight line using the least squares method. For this, we must linearize the results obtained in Fig. 7 and Fig. 8, taking decimal logarithms in the Eq. (3) in which it is obtained:

$$
\log B=\log \left(\frac{\mu_{0} m}{2 \pi}\right)+n \log (x)
$$

where $n$ is the exponent of $x$ and whose theoretical value as we know must be -3 . In this way, we already have a linear equation with which we can work through the method of least squares. If we represent $\log (B)$ versus $\log (x)$, we can obtain information on both the exponent of $x$ and the module of the magnetic moment as well as their absolute errors, through the slope and intercept at the origin, respectively, as shown in Table I. As we can observe, the magnetic moment, $m$, of each magnet is different, but the exponent of $x$, that is, $n$, in all cases except the refrigerator magnet 1 , is compatible with the expected theoretically (a value near to -3 ), being the discrepancy of only $0.6 \%$.

Table I. Experimental results of the value of the exponent of $x$ and the module of the magnetic moment of the magnet, after the corresponding adjustment by least squares to the four magnets used in this practice and whose graphics appear in Fig. 7 and 8.

\begin{tabular}{|c|c|c|}
\cline { 2 - 3 } \multicolumn{1}{c|}{} & $\boldsymbol{n} \pm \boldsymbol{\varepsilon}_{\boldsymbol{a}}(\boldsymbol{n})$ & $\begin{array}{c}\boldsymbol{m} \pm \boldsymbol{\varepsilon}_{\boldsymbol{a}}(\boldsymbol{m}) \\
\left(\boldsymbol{A} \cdot \boldsymbol{m}^{\mathbf{2}}\right)\end{array}$ \\
\hline Refrigerator Magnet 1 & $-3.17 \pm 0.15$ & $0.056 \pm 0.019$ \\
\hline Refrigerator Magnet 2 & $-3.10 \pm 0.13$ & $0.17 \pm 0.05$ \\
\hline Sphere Magnet & $-2.91 \pm 0.09$ & $1.6 \pm 0.3$ \\
\hline Magnet Ring & $-3.02 \pm 0.04$ & $1.29 \pm 0.12$ \\
\hline
\end{tabular}

Another observation that we could make is that refrigerator magnets have a magnetic moment between $3 \%$ and $10 \%$ of the magnetic moment of the two most powerful magnets that we considered, the sphere and the ring. 
E. Arribas, I. Escobar, C. P. Suárez, A. Najera and A. Beléndez, "Measurement of the magnetic field of small magnets with a smartphone: a very economical laboratory practice for introductory physics courses", European Journal of Physics Vol. 36, No. 6, Art. ID 065002, 11 pp (2015).

\section{CONCLUSIONS}

There has been designed a simple and very inexpensive laboratory practice for the laboratory of an introductory physics course for any grade of Sciences and Engineering. With the help of the magnetic sensor of the smartphones and a suitable app, the students are capable of determining, the dependency with the distance of the magnetic field, produced by different magnets.

Students can also make a graph and deduce from the experimental information in a relatively simple form that the magnetic field is inversely proportional to the distance to the cube. Also, through an adjustment by least squares it is possible to deduce the magnetic moment of every magnet with a good precision.

Using the magnetic sensor of the smartphones, it is possible to determine how the magnetic field of the small magnets changes with the distance. The experiment was made by the pupils of the first year of the major of Computer Engineering in the University of Castilla-La Mancha. Before the achievement of the experiment, the theoretical concepts related to the magnetic field produced by a magnet were studied. The experiment allowed the students to understand the importance of the distance in measuring the magnetic field and also to be comfortable with experiment designs. As it has been demonstrated, the results are completely in agreement with the theoretical prediction. Finally, and with the help of the suitable app, we think that the smartphones could be used for the achievement of laboratory practices in other fields of Physics.

\section{ACKNOWLEDGEMENTS}

One of us (AB) is very grateful to the Vicerectorship of Information Technologies of the University of Alicante (Spain) the help of GITTE-09006-UA. (IE) is grateful to the Ministry of Economy and Competitiveness the help provided through the project DPI2012-32994

\section{REFERENCES}

[1] M. Cole, Cognitive development and formal schooling: The evidence from cross-cultural research. L.C. Moll (editor), Vygotski and education: Instructional implications and applications of sociohistorical psychology. (Cambridge University Press, Cambridge UK, 1990).

[2] E. Bodrova and D.J. Leong, Herramientas de la mente: El aprendizaje en la infancia desde la perspectiva de Vygotsky. (Pearson Educación, México DF, 2004).

[3] J.A. Monsoriu, M.H. Giménez, J. Riera and A. Vidaurre, Eur. J. Phys. 26, 1149 (2005).

[4] S. Shamin, W. Zia and M.S. Anwar, Am. J. Phys. 78, 433 (2010). 
E. Arribas, I. Escobar, C. P. Suárez, A. Najera and A. Beléndez, "Measurement of the magnetic field of small magnets with a smartphone: a very economical laboratory practice for introductory physics courses", European Journal of Physics Vol. 36, No. 6, Art. ID 065002, 11 pp (2015).

[5] H.C. Chung, J. Liang, S. Kushiyama and M. Shinozuka, Int. J. Non-Linear Mech. 39, 691 (2004).

[6] T. Greczylo and E. Debowska, Eur. J. Phys. 23, 441 (2002).

[7] C. Sirisathitkul, P. Glawtanong, T. Eadkong and Y. Sirisathitkul, Rev. Bras. Ensino Fis. 35, 1504 (2013).

[8] O.O. Romulo and K.N. Franklin, Am. J. Phys. 65, 1115 (1997).

[9] T.W. Ng and K.T. Ang, Am. J. Phys. 73, 793 (2005).

[10] S.L. Tomarken et al., Am. J. Phys. 80, 351 (2012).

[11] M. Vannoni and S. Straulino, Eur. J. Phys. 28, 781 (2007).

[12] A. Kawam and M. Kouh, Phys. Teach. 49, 508 (2011).

[13] R. Ochoa, F.G. Rooney and W.J. Somers, Phys. Teach. 49, 16 (2011).

[14] J. Breitbart and G. Khanna, Numerical Analysis and Applied Mathematics, 1168, 1065 (2009).

[15] P. Vogt and J. Kuhn, Phys. Teach. 50, 439 (2012).

[16] J. Kuhn and P. Vogt, Phys. Teach. 50, 504 (2012).

[17] J.C. Castro-Palacio, L. Velazquez-Abad, M.H. Gimenez and J.A. Monsoriu, Am. J. Phys. 81, 472 (2013).

[18] J.C. Castro-Palacio, L. Velazquez-Abad, F. Gimenez and J. A Monsoriu, Eur. J. Phys. 34, 737 (2013).

[19] J.C. Castro-Palacio, L. Velazquez, J.A. Gómez Tejedor, F.J. Manjón and J.A. Monsoriu, Rev. Bras. Ensino Fis. 36, 2315 (2014).

[20] L.P. Vieira, V.O.M. Lara and D.F. Amaral, Rev. Bras. Ensino Fis. 36, 3505 (2014)

[21] H.D. Young and R.A. Freedman, University Physics with Modern Physics Technology Update, 13th edition. (Pearson Education, Inc., Naucalpan de Juarez, Mexico, 2012 chapter 21).

[22] W. Bauer and G.D. Westfall, University Physics with Modern Physics. (The McGraw-Hill Companies, Mexico DF, Inc., 2011 chapter 22).

[23] J.A. Manzanares, J. Bisquert, G. Garcia-Belmonte and M. Fernandez-Alonso, Am. J. Phys. 62, 702 (1994).

[24] A. Franco, http://www.sc.ehu.es/sbweb/fisica/elecmagnet/elecmagnet.htm . This website was last visited 2015-1-6.

[25] This app can be downloaded from the following link: https://itunes.apple.com/es/app/magnetometer/id342782714? $\mathrm{mt}=8$. This website was last visited 2014-12-31. 
E. Arribas, I. Escobar, C. P. Suárez, A. Najera and A. Beléndez, "Measurement of the magnetic field of small magnets with a smartphone: a very economical laboratory practice for introductory physics courses", European Journal of Physics Vol. 36, No. 6, Art. ID 065002, 11 pp (2015).

[26] This app can be downloaded from the following link:

https://play.google.com/store/apps/details?id=com.chrystianvieyra.android.physicstoolboxmag netometer\&hl=es . This website was last visited 2014-12-31. 